\title{
What is stereoscopic vision good for?
}

Jenny C. A. Read

Jenny C. A. Read, "What is stereoscopic vision good for?," Proc. SPIE 9391, Stereoscopic Displays and Applications XXVI, 93910N (17 March 2015); doi: $10.1117 / 12.2184988$

SPIE Event: SPIE/IS\&T Electronic Imaging, 2015, San Francisco, California, United States 


\title{
What is stereoscopic vision good for?
}

\author{
Jenny C. A. Read \\ Institute of Neuroscience \\ Newcastle University
}

\begin{abstract}
Stereo vision is a resource-intensive process. Nevertheless, it has evolved in many animals including mammals, birds, amphibians and insects. It must therefore convey significant fitness benefits. It is often assumed that the main benefit is improved accuracy of depth judgments, but camouflage breaking may be as important, particularly in predatory animals.

In humans, for the last 150 years, stereo vision has been turned to a new use: helping us reproduce visual reality for artistic purposes. By recreating the different views of a scene seen by the two eyes, stereo achieves unprecedented levels of realism. However, it also has some unexpected effects on viewer experience. The disruption of established mechanisms for interpreting pictures may be one reason why some viewers find stereoscopic content disturbing.

Stereo vision also has uses in ophthalmology. Clinical stereoacuity tests are used in the management of conditions such as strabismus and amblyopia as well as vision screening. Stereoacuity can reveal the effectiveness of therapy and even predict long-term outcomes post surgery. Yet current clinical stereo tests fall far short of the accuracy and precision achievable in the lab. At Newcastle University, we are exploiting the recent availability of autostereo 3D tablet computers to design a clinical stereotest app in the form of a game suitable for young children. Our goal is to enable quick, accurate and precise stereoacuity measures which will enable clinicians to obtain better outcomes for children with visual disorders.
\end{abstract}

Keywords: stereoscopic vision, visual neuroscience, binocular vision, visual cortex, evolution, 3D displays, picture perception

\section{INTRODUCTION}

Stereoscopic vision has been justly described as "one of the glories of nature" ${ }^{1}$. Humans can detect disparities between the two eyes' images which are less than the diameter of one photoreceptor. But there are many other ways of reconstructing 3D depth information other than stereoscopic vision. When we close one eye, the most obvious change is the loss of peripheral vision on one side, rather than any alteration in perceived depth. Indeed, many people are stereoblind without even realising it. So what is stereoscopic vision actually good for? In this review, I will consider some of the uses stereo vision has been put to in three different domains: in nature, in art and in medicine.

By the rather loose term "nature", I mean our evolutionary history and animals other than Homo sapiens. I will consider how stereopsis may improve an animal's fitness and thus have been selected for during evolution. In "art", I will consider how stereo displays can help us get closer to reproducing reality, but also why this may bring its own problems. In "medicine", I will consider the uses and limitations of stereoacuity measurements in binocular vision disorders. In each case, my discussion will be partial and incomplete, leaning heavily on my own interests and areas of research.

Stereoscopic Displays and Applications XXVI, edited by Nicolas S. Holliman, Andrew J. Woods,

Gregg E. Favalora, Takashi Kawai, Proc. of SPIE-IS\&T Electronic Imaging, Vol. 9391, 93910N

(C) The Author(s). Published under a Creative Commons Attribution CC-BY 3.0 License · doi: 10.1117/12.2184988 


\section{WHAT IS STEREOSCOPIC VISION GOOD FOR IN NATURE?}

Why did stereoscopic vision evolve? The answer seems obvious: to detect depth. But in fact, there are many ways to detect depth. We can judge an object's distance by seeing how it appears to move when we move our head (motion parallax), or by measuring the focal distance we need to bring a blurred object into sharp focus (accommodation). These examples are even geometrically equivalent to stereopsis, since all three effectively work by triangulating back from two different lines of sight to an object: from left and right eyes, from position 1 and position 2, or from diametrically opposed edges of the pupil ${ }^{2,3}$. Other depth cues include perspective, relative size, occlusion, shading / shadows, texture, aerial perspective. Admittedly all these cues have their own disadvantages. Motion parallax requires you to move, potentially alerting prey to your presence. Blur cues are not signed. Perspective cues are fundamentally ambiguous and require a host of assumptions to interpret. But stereo vision has significant disadvantages too.

First, for animals no less than for makers of 3D movies, stereo is expensive. Most obviously, it requires duplication: two eyes must scan the same region of visual space. This necessarily reduces resources elsewhere. For humans, the cost is obvious: given that we have two eyes each with a visual field of about $70^{\circ}$, we sacrifice vision behind us by placing both eyes on the front of our head. Binocular vision doesn't necessarily require an animal to sacrifice all-round vision in this way. For example, the praying mantis has two compound eyes cover almost the entire visual field while including a substantial region of binocular overlap which allows them to use stereo vision. However, the mantis has made sacrifices too: its visual acuity is limited by the finite number of facets (ommatidia) in its eyes, so by directing two ommatidia to the same point in space, it loses resolution.

Admittedly, binocular vision brings advantages which are in principle independent of stereopsis. Until the discovery of stereopsis, scientists assumed the main advantages of human's binocular vision was redundancy. Even if we lose an eye, we can still see the critical visual field directly in front of us; the loss of vision is mainly in the periphery. More subtly, having two visual sensors covering the same region of the visual field enables a better signal to noise ratio; humans are generally better at detecting weak visual signals if viewing them with both eyes rather than just one ${ }^{4}$. Recently, Changizi \& Shimojo ${ }^{5}$ drew attention to the "X-ray vision" offered by binocular vision. When two eyes view the same region of space from different viewpoints, objects that are hidden from one eye may be visible to the other. This may make binocular vision particularly valuable for species that live in visually cluttered habitats like leafy forests. Interestingly, such environments are also cited by cinema stereographers as a strength of 3D movies: viewers can interpret cluttered scenes better in $3 \mathrm{D}$ than in conventional $2 \mathrm{D}$ movies.

Once binocular vision has evolved, a species has to have some mechanism for fusing the two eyes. In a species with fixed eyes which viewed only distant objects, this could be as easy as summing output from photoreceptors pointing in the same visual direction. However, for most animals, life is not so simple. Matching up the two eyes' images is a computational challenge known as the stereo correspondence problem ${ }^{6}$. It is greatly simplified if the relative position of the eyes is known. Then, we need only consider epipolar matches ${ }^{7}$. Machine stereo algorithms are often able to restrict their analysis to this case ${ }^{8}$.

In animals like humans with mobile eyes, stereo correspondence is in principle two-dimensional - an object projecting to a given point in the left retina could in principle project anywhere in a $2 \mathrm{D}$ patch of the right retina, depending on where the person is looking ${ }^{9}$. In practice, the human visual system tries to "fudge" stereo correspondence into a 1D problem by imposing tight constraints on binocular eye movements ${ }^{10}$. Our eyes certainly cannot look independently in different directions like those of the chameleon! Even so, the epipolar lines rotate on the retina as our eyes move, so that different combinations of horizontal and vertical disparity become possible. In a computer, a stereo algorithm might loop through all these different possible matches. In brains, different possible matches appear to be considered in parallel, each one by a different binocular neuron (brain cell) which receives input from the appropriate points on left and right retina. 
Apparently, humans do not have enough neurons to account for all the matches which are possible given the range of our eye movements. As a consequence, our stereo vision doesn't work for unusual gaze postures, e.g. if we are looking steeply up or down ${ }^{11}$.

As Martin \& Katzir ${ }^{12}$ point out, it's not safe to assume that any species that has evolved a binocular overlap has necessarily also evolved stereoscopic depth perception. However, given that the binocular images have to be matched up anyway, some form of stereopsis would appear to be a relatively inexpensive add-on to binocular vision. So what is this stereopsis good for?

Interestingly, all the non-mammalian species known to have stereo vision have eyes which are either fixed in place on the head (the praying mantis ${ }^{13}$ ) or have very little mobility (e.g. toads ${ }^{14}$, owls ${ }^{15}$, falcons ${ }^{16}$ ). Stereo correspondence for these species genuinely is a one-dimensional problem. And with fixed eyes, once you know the binocular disparity of an object, you also automatically know its distance. For these hunting species, stereopsis offers a robust range-finding service to guide their predatory strikes.

We have already seen that mobile eyes complicate stereo correspondence. Worse, eye movements mean that there is no unique mapping between retinal disparity and depth. Two objects which differ in disparity by $0.5^{\circ}$ could be $12 \mathrm{~cm}$ apart or $6 \mathrm{~m}$, depending whether the fixation point is $1 \mathrm{~m}$ or $10 \mathrm{~m}$ away. Humans do have some ability to make these corrections, but we aren't very accurate, so our ability to detect absolute distance via stereopsis is rather poor. Really, our stereo vision is good for revealing relative depth ${ }^{17}$. This is advantageous for makers of $3 \mathrm{D}$ content, as it means we are unlikely to perceive even substantial distortions in absolute disparity. The relative disparity cues will give us a satisfyingly vivid impression of relative depth, and we will probably not notice that the disparity indicates the basketball is an ellipsoid rather than a sphere.

For humans, stereo vision seems to be particularly useful in visually-guided manual tasks ${ }^{18-20}$, perhaps especially when using tools, since the tool itself does not provide sensory feedback ${ }^{21}$. Trying to thread a needle with one eye closed is a good way to appreciate the value of relative depth information from stereopsis.

Remarkably, stereo vision continues to reveal relative depth structure even when this is not otherwise visible. This has been exploited for military purposes since the First World War. In his 1920 book “Aerial Photography”, Herbert Ives ${ }^{22}$ describes two things stereo photography is particularly good for. First, "to detect undulations of ground". Except where slanting sunlight produces shadows, aerial photographs generally look flat, so that "small local elevations and depressions cannot be distinguished from mere difference in color or marking. But with stereoscopic views these features stand out in a striking manner." Second, to reveal depth edges which may indicate militarily significant targets or obstacles. "Once presented in relief, camouflaged buildings or gun emplacements, however carefully painted, are ridiculously easy to pick out."

This camouflage-breaking property of stereopsis is demonstrated beautifully in the random-noise stereograms created by Béla Julesz ${ }^{23}$. A similar effect is used in the "Magic Eye"-style autostereograms ${ }^{24}$ where a 3D shape is revealed when the eyes are crossed so that the left and right eyes view different parts of the image.

Camouflage is a critical defence of many prey species, so the evolutionary advantages of being able to overcome it are obvious. Julesz ${ }^{23}$ suggested that this camouflage-breaking ability might be the major reason stereo vision evolved. In barn owls, it might reveal the 3D structure of a mouse camouflaged against a woodland floor; in monkeys, it might reveal a tasty insect camouflaged against bark.

Stereopsis is believed to have evolved independently in mammals, birds and insects ${ }^{25}$. It is possible it has evolved more often than that, although we do not yet know enough about stereo abilities in different animals to be sure. Stereopsis has presumably been selected for different purposes in different species, so one would expect it to have different properties. 
For example, it is generally accepted that stereopsis in insects must be much simpler than in a complex organism such as ourselves ${ }^{26}$. Praying mantises have around a million neurons, and this together with the simplification afforded by their fixed eyes is demonstrably enough to support a stereo vision system capable of detecting whether a prey item is within strike range. It is not yet known whether mantis stereo vision can also break camouflage. Owls and humans are both binocular predators, but have very different lifestyle, habitat, anatomy and visual systems, so one might have expected their stereo vision to be correspondingly different. Strikingly however, owl stereopsis, which has been studied in some detail, appears to be qualitatively identical to our own ${ }^{15,27,28}$.

It used to be thought that stereo vision was found only in visually-guided predators with front-facing eyes, such as humans and cats. However, we now know that herbivores with lateral eyes can have stereo vision too. Neurons which appear to support stereopsis are found in several such animals, e.g. sheep ${ }^{29}$, and camouflage-breaking stereopsis has been demonstrated behaviourally in the horse ${ }^{30}$. Very little is known about what stereo vision is good for in these species. Many of its proposed uses in other species - predation, manual dexterity, tool use, jumping accurately from branch to branch ${ }^{31}$ - hardly apply to these species. Camouflage breaking may be useful if it helps reveal a lurking predator. We have already seen that stereo vision can be useful for revealing unevenness in the ground plane ${ }^{22}$. Indeed, Helmholtz long ago noted that certain features of human stereo vision may indicate that it is optimized for depth perception in the ground plane ${ }^{32,33}$, and there is evidence that binocular viewing aids locomotion ${ }^{34}$. Stereo vision may also be valuable in these species because it helps them run at speed over rough ground without stumbling. Perhaps the proverbial agility of the mountain goat is at least partly supported by its disparity-tuned neurons ${ }^{29}$

Finally, one advantage which stereo vision may offer to many species is that depth structure indicated by stereopsis is hard to fake. Other visual cues such as color, brightness and motion have been exploited by evolution to offer deceptive signals. Animals can use patterns of color and luminance to mimic a more threatening species, or to blend into the background and evade notice altogether. They can conceal their movement; a nice example is the hoverfly, which flies directly along the line of sight to its intended prey ${ }^{35}$. Even perspective cues can be manipulated, e.g. by bowerbirds who fake projection geometry to make their bowers appear larger than they really are ${ }^{36}$. However, I cannot think of any example where a non-human animal is able to manipulate stereo depth cues in order to make its size, distance or structure appear other than it really is. This reliability must be a key advantage of stereo vision in nature.

\section{WHAT IS STEREOSCOPIC VISION GOOD FOR IN ART?}

All that changed for humans in 1838, when Charles Wheatstone demonstrated the "remarkable, and hitherto unobserved" phenomenon that a compelling illusion of depth could be produced by arranging for the two eyes to see different images, each drawn from the appropriate viewpoint ${ }^{37}$. The discovery of stereopsis came at an interesting time. Reproducing reality - creating a visual world for the observer which differs as little as possible from a real scene - had been an obsession of Western art for thousands of years ${ }^{38} .{ }^{39}$ Pliny the Elder ${ }^{40}$ (ch XXXV) describes a competition between the artists Zeuxis and Parrhasius, where Zeuxis painted grapes so real that the very birds flew down to pick them. (Zeuxis then demanded Parrhasius draw aside the curtain covering his entry, only to discover that he had been fooled by a masterpiece of trompe l'oeuil - the curtain was Parrhasius' painting.)

The pinhole camera or camera obscura demonstrated that it was in principle possible to recreate a real visual scene on a flat surface ${ }^{41}$. The development of perspective was a powerful new tool to this end. Yet binocular vision was always recognised as a fundamental problem. A camera obscura, or a mirror, offers correct views to both eyes. A painting can only ever be perfect for one viewpoint ${ }^{42}$. Thus, as Leonardo lamented ${ }^{42}$ (chap. CCCXLVIII) "If nature is seen with two eyes, it will be impossible to imitate it upon a picture so as to appear with the same relief, though the lines, the lights, shades, and colour, be perfectly imitated." A low-tech way of overcoming this problem was to require people to view with one eye (chap. CXXIV). Brunelleschi exploited this with his famous "peepshow": a perspective painting of Florence, designed to be viewed with one eye via a mirror ${ }^{43}$. Without binocular cues to flatness, and with the mirror surface further disrupting cues to the true picture plane, the perspective/shading depth cues dominate and the painting 
appears in depth. This effect was dubbed "monocular stereoscopy" by Claparède ${ }^{44}$. Wheatstone's true "binocular stereoscopy" went further, not just removing the stereo cues indicating flatness, but simulating stereo cues to depth. It is amusing to speculate how Leonardo, Raphael or van Hoogstraten would have reacted if Wheatstone's discovery had come a few centuries earlier. Would they have had the mastery to paint binocular pairs of masterpieces which could be viewed in a stereoscope?

The question never arose, because just a year after Wheatstone's presentation to the Royal Society, Louis Daguerre's process was published, using "light itself [to] reproduce the shapes and proportions of external objects, with almost mathematical precision" ${ }^{45}$. The new science of photography rapidly overtook painting in the accurate reproduction of the information visible to a single eye. Stereoscopy enabled photographers to reproduce and display both eyes' views, amazing nineteenth-century audiences with the vividness of the illusory depth. Fine art now largely abandoned its historic attempt to reproduce reality, at least in the optical sense. The modern heirs of Leonardo and Raphael are computer graphics and television engineers, who devote years to mastering the play of light in a scene and to reproducing it correctly. Unlike the artists of old, they are not forced to try and capture an event in a single arrested image ${ }^{46}$, but accurately rendering and reproducing movement brings its own challenges. Recent years have seen many further innovations aimed at overcoming the artefacts which distinguish art from reality. These include high definition displays to remove visible spatial artefacts such as pixelation; high frame rates to remove temporal artefacts such as motion blur and judder; high dynamic range to move closer to the billion-fold range of light intensities over which human visual systems operate; and wider color gamuts to move close to the full range of discriminable colors. And it's not just vision: better sound systems are continually being developed, while some theme-park-style attractions even stimulate the vestibular and somatosensory systems (e.g. http://www.simworx.co.uk/).

Opinion is divided as to whether the quest to reproduce reality is worthwhile. In the motion picture industry, it is usually justified on the grounds that it will increase audience immersion, supporting and sustaining the illusion that we are in the world created by the director ${ }^{47}$ : "a sort of man-made dream for those who are awake" ${ }^{48}$. Yet innovations such as high frame rate and stereoscopic 3D are not always welcomed by audiences and critics ${ }^{49}$. Sometimes, this is because we like seeing what we are used to seeing; for example, audiences expecting wagon wheels to turn backwards in Westerns. Sometimes, problems are due to the limitations of technology. A good example is the much-studied "vergence/accommodation conflict" in stereo displays. When humans view real scenes, we direct (converge) both eyes at the object of interest and also focus our eyes (accommodate) on it. On a 2D display, viewers as usual have to converge and focus on the same object: the screen. But when stereoscopic 3D simulates an object substantially away from the screen plane, viewers have to converge and focus at different distances. It is well documented that this can cause viewers discomfort and eye fatigue ${ }^{50-53}$. Such issues can be avoided by ensuring that the conflict is kept within the "comfort zone" 54 , and, ultimately, overcome with better technology, e.g. multiple viewpoint displays. But coming closer to reality can bring its own problems.

In my lab we've recently been investigating one example: the issue of oblique viewing. It has been known ever since invention of perspective that geometrically, perspective only "works" from one viewpoint. For example, the perspective master Andrea Pozzo painted the ceiling of St Ignatius' cathedral in Rome so as to simulate additional vaults and columns soaring above the actual roof of the church $\left({ }^{55}\right.$, Figure 2). To achieve the desired effect, you have to stand so your eye is at the centre of projection. This position is helpfully marked on the floor of the cathedral by a yellow disk. Apparently, the effect is highly compelling. Away from this place the architecture looks distorted, as if about to collapse in on the viewer ${ }^{56}$.

Yet in fact, Pozzo's ceiling is a highly unusual example. In our everyday experience, perspective pictures or photographs generally don't appear distorted if we view them from the "wrong" place .Admittedly one reason is simply because we are quite insensitive to such distortions ${ }^{57}$. Yet it is also clear that part of it is active "compensation" for the oblique viewing. That is, if we can recognise an object as a picture and detect the picture plane, then our brains somehow correct 
for the oblique viewing and perceive the picture as if we are viewing it front on. We can see the effect of this compensation by looking at photographs of photographs, where the latter is shot from an oblique angle. We correct for the picture plane of the second photo, not for that of the photographed photo.

It is an interesting question to what extent such correction for oblique viewing is learnt or innate. Vishwanath et al ${ }^{58}$ suggest that it is a side-effect of mechanisms which evolved to allow us to perceive real objects. For example, we recognise that the rim of a coffee cup forms a circle in space, even if it projects to an ellipse on our retina. Pizlo ${ }^{59}$ has argued that the symmetry of the cup may be critical in such shape judgments.

In compensating for oblique viewing, perceiving the picture plane is clearly critical. One of the reasons Pozzo's ceiling is both so compelling when seen from the correct viewpoint, yet appears distorted from the wrong viewpoint, is probably to do with the difficulty of detecting the picture plane. The picture is far enough away that binocular disparity does not obviously signal that the simulated architecture is in fact two-dimensional. Additionally, there is no "picture plane", since the architecture is painted on a hemicylindrical ceiling. Normally when we view a picture, there are many cues to the orientation of the picture's surface: perspective cues from the edge of the picture, accommodation cues and so on. In 2D displays, binocular disparity is probably one of the most reliable. By disrupting this, stereo displays may reduce our ability to detect the picture plane; indeed Pirenne ${ }^{56}$ claimed that "In [stereoscopic images], the observer is hardly aware of the surface of the picture, as a surface." This, it is argued, reduces our ability to compensate for oblique viewing. Accordingly, when we move back \& forth in front of a stereoscopic image, we see the object rotate to keep facing us. Of course, 2D pictures also appear to keep facing us as we move. A famous example is the "Uncle Sam needs YOU" poster. The finger is pointing directly at us only if we are positioned directly in front of the poster, yet we perceive the finger pointing at us no matter where we stand.

There has been surprisingly little study of the extent to which stereo displays disrupt viewers' ability to compensate for oblique viewing ${ }^{60-62}$. Potentially, this could contribute to the audience resistance to 3D media. Most viewers in a cinema, and many at home, will be viewing the screen obliquely. If as a result objects appear subtly distorted, whether or not viewers consciously notice this, it could make them feel somehow ill at ease.

We have been examining this by showing viewers rendered images of cubes (Figure 3). In our experiments, observers were shown two cubes, one above the other on a passive stereo 3D monitor. One cube was rendered in the normal way (Figure 3A). The other was rendered for oblique viewing, like taking a photo with the film tilted relative to the lens (Figure 3B-E). We asked observers to report which cube appears more like a cube, in the sense of having parallel sides of equal length, square faces and so on. Sometimes, the observer was viewing the screen as usual, with it straight on in front of them. But sometimes, it was tilted away from them so they were actually viewing it from one side.

Geometrically, in this case, the cube should look best when rendered for the actual oblique viewing angle. So the cube in Figure 3E should in theory look less distorted than the cube in Figure 3A, if you view it with one eye from an angle of $45^{\circ}$ to the page. But if viewers successfully compensate for oblique viewing, they'll prefer the normally-rendered cube, even if the image on the retina is distorted. Sure enough, with normal 2D viewing we found that the normally-rendered cube was preferred almost all the time, even when viewers were viewing the screen obliquely. Only at the most oblique viewing angle, $45^{\circ}$, was the geometrically correct cube chosen significantly more often than the normal cube, and even then not overwhelmingly so, indicating that viewers had no very clear preference. Surprisingly, it made relatively little difference whether the edges of the screen were visible (so observers could see they were viewing it obliquely) or hidden, whether the cubes were static or rotating, or whether the cubes were presented in $2 \mathrm{D}$ or $3 \mathrm{D}$, although in each case the latter option did weaken the compensation. That is, the geometrically-correct cube was more likely to be chosen when the cubes were 3D, rotating and the edges of the screen were hidden. Importantly for this paper, 3D had the greatest effect: that is, as predicted, stereo displays really do weaken the extent to which viewers compensate for oblique 
viewing. But stereo does not abolish compensation; even in 3D, our results indicated that perceptual compensation for oblique viewing still operated.

It is not clear whether our choice of cubes was critical here. Banks et al. ran a similar experiment using a different stimulus, task and viewing distance, and concluded that compensation for oblique viewing was almost completely absent for stereoscopic stimuli. Further work is needed to understand what aspects of the experiment are critical. In the meantime, our work indicates that, despite technical limitations, current stereo displays already work well enough for most people.

The place of stereoscopy in art is still disputed. I have argued that the distinctive contribution of stereoscopy is to improve our ability to reproduce reality. As we saw in the previous section, stereopsis is perhaps uniquely veridical: it rarely fails in nature, and is virtually impossible to fake other than by human artifice. For this reason, stereoscopic displays should be uniquely capable of convincing audiences that they are viewing a real scene. However, this is not the only or even the main goal of art. Excessive realism may prove disconcerting for viewers or be dismissed as a gimmick ${ }^{43}$ (chapter 5). Perhaps for this reason, stereoscopic media is used much more often in entertainment than in fine art, and even in entertainment, it is sometimes criticized as a theme-park effect. However, the visual media industries show no sign of abandoning the age-old quest for ever greater realism. In the domain of stereoscopy, glasses-free 3D, virtual reality head-sets and multi-parallax displays are some of the technologies under development. It will be interesting to see how artists in film, video games and other industries exploit these new opportunities.

\section{WHAT IS STEREOSCOPIC VISION GOOD FOR IN MEDICINE?}

The desire to create a realistic reproduction of a virtual 3D scene is not limited to entertainers. The fantasy world which is displayed to observers may not be an alien planet, but a set of abstract data, or the inside of a human body. Accordingly, stereoscopic data visualisation has many applications in medicine, for example in radiography. Stereo displays can help train surgeons on simulators, or aid them during real operations. But here I will restrict myself to an area where I have particular expertise, and talk about the uses of stereo vision in assessing binocular visual function.

As discussed above, stereopsis is an extremely exacting ability for any animal. To function, it requires all components of the visual system to be working well - the two eyes, including their optics and retina; the optic nerves which convey the images to the brain; the brain circuitry which compares and contrasts the images from the two eyes; and in humans, also highly precise control and coordination of eye movements. When this is all working correctly, human stereo vision is astonishingly precise. This is conventionally assessed by measuring the smallest difference in the depth of two adjacent surfaces which can be detected by stereo vision. This distance is conventionally expressed as visual angle (e.g. degrees) rather than as a physical distance (e.g. in metres), since the distance depends on the distance to the surfaces in question: it's easier to detect a $1 \mathrm{~mm}$ depth step between two surfaces at $60 \mathrm{~mm}$ and $61 \mathrm{~mm}$ from the observer than the thousand-fold greater step between two surfaces at $60 \mathrm{~m}$ and $61 \mathrm{~m}$. In contrast, an individual's stereoacuity is roughly constant when expressed as visual angle. In humans, stereoacuity can be as low as 2 seconds of arc, or just $0.0006^{\circ}$.

It is not surprising that such a subtle ability is prone to problems. Approximately $2 \%$ of children have some form of strabismus ${ }^{63}$, i.e. their eyes are incorrectly aligned. There are many different forms of strabismus. In infantile esotropia, which develops within the first two years of life, the eyes are constantly turned inwards (crossed). In intermittent exotropia, which generally manifests itself in older children, the misalignment is not constant, but shows up mainly when the child is tired or looking at objects far away. In this condition, one eye turns outwards, so that the two eyes' lines of sight diverge instead of remaining parallel or converged. These conditions either resolve or (more often) are corrected surgically during childhood, so that patients under treatment are usually children.

Normally, we direct both eyes at the object we want to view, but people with strabismus are unable to do this. They have to fixate objects with one eye at a time. Some people develop a strategy of "alternating fixation": viewing sometimes with the left eye and sometimes with the right. Others always use the same eye. Because the eyes are viewing different 
objects, the brain has to suppress the unwanted image from consciousness. This is something we can all do to some extent ${ }^{64}$. For example, we've probably all had the experience of sitting at the back of an auditorium in a position where only one of our eyes can see the speaker, the other viewing the back of someone's head. Generally, our brains manage to prevent the unwanted head from obtruding too much on our visual perception. However, if one eye's image is suppressed all the time, gradually the brain will reduce its response to visual input in that eye. The result is a long-term loss of vision in that eye. This condition is amblyopia, sometimes picturesquely called "lazy eye". Despite the name, the loss of vision is not caused by any problem with the eye's optics or retina, but by a loss of neurons dedicated to processing its image. Strabismus is not the only cause of amblyopia. It can also develop if the two eyes have very different refractive errors (anisometropia), so that one eye's retinal image is blurrier than the other. Again, the brain learns to ignore input from the eye with the poor-quality image. Both strabismic and anisometropic amblyopia can persist even after the original cause of the problem has been corrected, e.g. the eyes have been aligned surgically or spectacles have been prescribed. The prevalence of amblyopia is around $1.3 \%$ in the USA ${ }^{63}$.

Stereoacuity measurements play an important role in the management of such patients. In a recent review, Elliot \& Shafiq ${ }^{65}$ note that "a wide range of tests exist to diagnose the presence and the quality of binocular vision", but state "a measurement of stereoacuity [is] considered the 'gold standard'." In clinical studies of the effectiveness of different treatments, stereoacuity measurements are a critical outcome measure. For example, ${ }^{66}$ showed that the number of amblyopic children with detectable stereo vision rose from $34 \%$ before treatment to $57 \%$ afterwards. ${ }^{67}$ showed that playing a specially-designed computer game produced a significant improvement in stereoacuity. These improvements in stereo vision demonstrate that treatment has produced a clear improvement in binocular function.

Stereoacuity can also predict, not simply measure, therapeutic outcomes. In one study of children with esotropia, children who still had no stereo vision shortly after corrective eye surgery were 4 times more likely to require further surgery than children who appeared clinically similar before their first surgery, but had measurable stereopsis after their operation ${ }^{68}$.

In infantile esotropia, stereoacuity measurements have led doctors to argue for alignment surgery as soon as possible after diagnosis, to minimize the amount of time children view the world through misaligned eyes ${ }^{69,70}$. Babies are almost certain to develop stereo vision if their eyes are aligned before the age of six months, whereas if alignment is delayed until the child is 18 months, the child has a less than 1 in 5 chance of developing stereo vision ${ }^{71}$.

The effectiveness of stereopsis in screening for visual disorders, as opposed to monitoring treatment, is disputed. Nevertheless, most US authorities recommend that stereoacuity measurements should form part of pediatric visual screening ${ }^{72}$.

Stereoacuity is not only important as a way of measuring binocular vision function, it is also important in its own right. "Improved stereoacuity outcomes are associated with better long-term stability of alignment, reduced risk for and severity of amblyopia, improved achievement of sensorimotor developmental milestones, better reading ability, and improved long-term quality of life" ${ }^{73}$.

Stereoacuity is an example of a psychophysical threshold measurement. These measurements are inherently probabilistic. For example, consider the "threshold luminance" at which a lightbulb is "just bright enough for an individual to see." It is not the case that if we add one more photon, the person will always perceive the light, whereas if we block one photon, the person will never notice it. Rather, the person will spot the light on some proportion of trials, ranging from 0 to $100 \%$ as the light becomes more visible. Similarly, there is no unique depth step which is "just big enough to detect". The proportion defined as threshold is a matter of choice and convention. Vision scientists have developed rigorous techniques for making such threshold measurements. Due to the probabilistic nature of the measurement, these require many trials, just as one has to toss a coin many times in order to assess whether it is fair or 
biased. The tests are controlled by computers, which implement algorithms designed to be statistically optimal; i.e. to produce the most reliable estimate of threshold in the minimum number of trials.

However, the laboratory tests are not suitable for most clinical settings. They require bulky equipment: a computer to control the algorithm, often a mirror haploscope to display the stereoscopic images, a headrest in order to ensure that the subject remains at a fixed viewing distance. Clinicians use commercial stereotests which are much cruder. They do not exploit new stereo display technology, but rely on anaglyph or passive polarization stereo, lenticular stereo or physical depth. The latter two have the drawback of offering non-stereo cues if the patient moves relative to the test card. They come as a set of cards or plates with a very limited choice of disparities. Because the tests are run by a human being, not a computer, they cannot use complex algorithms. They typically start with large, easy disparities and run until the patient has made a mistake on, say, 2 out of 5 repeats with the same disparity. The tests are not particularly engaging for small children, who may also be unwilling to wear the 3D glasses necessary for many tests. If a child stops answering, it may not be clear whether this is because the disparity is now below their stereo threshold, or simply because they are bored of the task. Presumably for all the above reasons, clinical stereotests have poor reliability. A recent study concluded that for most tests, changes of less than a factor of 4 could not be distinguished from test-retest variability ${ }^{74}$. That is, if a child undergoing amblyopia therapy shows a stereoacuity of $200 \operatorname{arcsec}$ on one visit and of $50 \operatorname{arcsec}$ on the next visit, one cannot be sure whether this is real improvement or simply measurement error.

To address these problems, a multi-disciplinary team at Newcastle University has recently begun the ASTEROID "accurate stereotest" project. This team, which includes vision scientists, ophthalmologists, orthoptists and software engineers, is developing a stereotest which will run on the NEO3DO tablet computer (www.neo3do.com). This uses parallax-barrier technology to achieve glasses-free 3D. The task is to locate a target patch, defined by disparity to appear floating in depth in front of a background surface. The target and background are both constantly changing random patterns of colored dots, so the target is perfectly camouflaged without any edges to give away its location; the task can be solved reliably only by using stereo vision. By giving target and background equal and opposite screen parallax, so neither target nor background lies in the screen plane, we avoid non-stereo cues which could otherwise arise if the patient tilted the screen so as to make images intended for different eyes visible to both. Because the test is administered on a computer, we can use complex algorithms designed to be statistically optimal, e.g. adaptive Bayesian staircases ${ }^{75,76}$. The algorithm automatically accounts for the level of performance which is possible by chance.

We can also use the device's inbuilt front camera to monitor viewing distance. This is important, because the relationship between the physical parallax on the test device and the binocular disparity at the retina depends on viewing distance. In current clinical stereotests, the clinician has to ensure that the child performs the test at a constant distance, intervening if the child tries to lean forwards for a better view. We can simply correct for any changes in viewing distance.

Perhaps most importantly, the stereotest will be presented in game format, with a range of possible themes designed to appeal to children with different interests and ages. The child will give their answers via the device's touchscreen, touching the place where they perceive the disparate target. This is a simple and intuitive means which will be familiar to most modern children, and is likely to be more appealing to them than interacting with a strange adult. Traditional game mechanics are well suited to our needs, with easy early game-play becoming successively more challenging as the player goes up through the levels, very much as in a psychophysical staircase. Sound, color and animation will be used to maintain the child's engagement with the game and encourage them to complete many trials, enabling more accurate results. The game aspect of the ASTEROID project is being developed by Fluid Pixel (www.fluidpixel.com), a mobile software design company based close to Newcastle University. We are involving local children and patients wherever possible, e.g. in the design of attractive characters.

The ASTEROID project aims to exploit new stereo display technology to create a fun, convenient way for clinicians to obtain more reliable data about their patients' visual function, ultimately resulting in better treatment and outcomes. This 
is only one small example of how new 3D technology can be applied in medicine. There are already many other applications, and more will certainly arise in the future.

\section{CONCLUSION}

Initially, stereo vision was thought to be restricted to a small number of "higher" animals with large brains and frontallyplaced eyes. But we are gradually discovering it in more and more species, including several with lateral eyes and/or small brains. Despite its expense in terms of hardware and software, stereo vision has been selected for time and again throughout evolution. This suggests that the advantages it offers are profound. The uses of stereo vision likely differ between species, but include range-finding, relative depth detection and camouflage breaking. A distinctive advantage of stereo vision in evolution is that it is hard to fool. Humans are the only species who are able to present illusory stereo stimuli, i.e. to trick stereo vision systems into perceiving a depth structure which does not exist. This has enabled us to recreate visual scenes with unprecedented accuracy, although this is not been without problems of its own. With the advent of digital technology, humans are continuing to find new uses for stereo vision in fields such as entertainment, data visualisation and medicine.
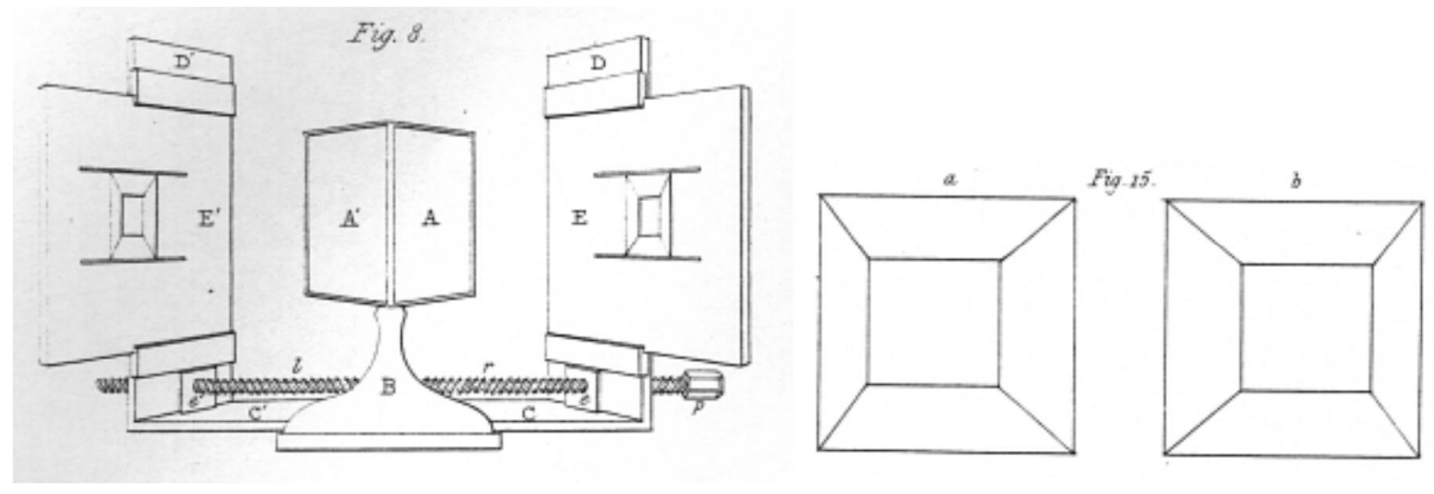

Figure 1. Wheatstone stereoscope and stereogram ${ }^{37}$ 


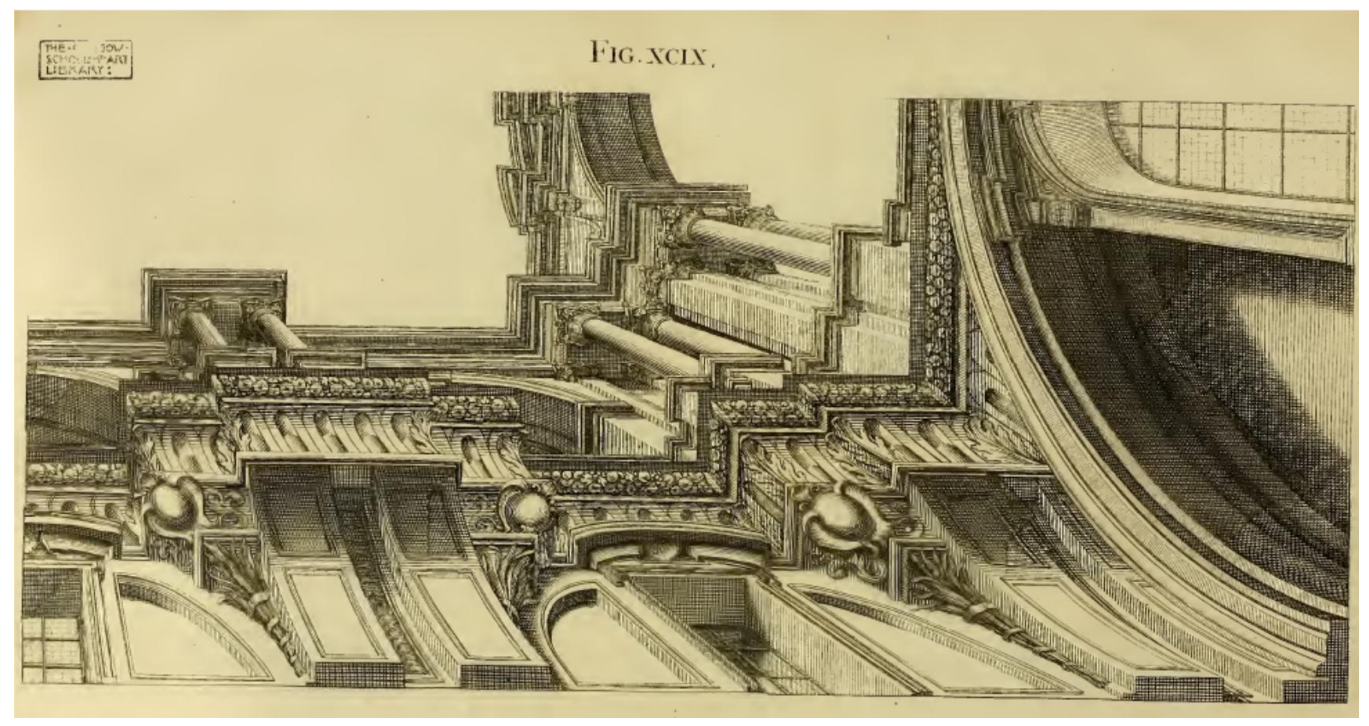

Figure 2. Pozzo's plan for the imaginary architecture rendered on the ceiling of St Ignatius; fig 99 of Pozzo ${ }^{55}$
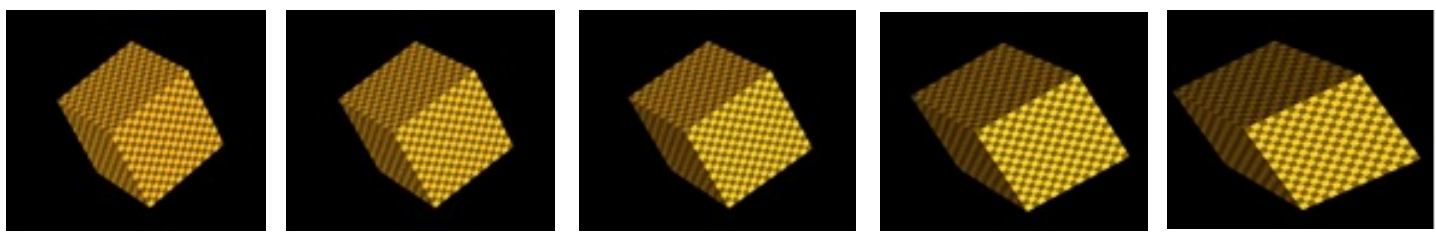

Figure 3. Examples of our "cube" stimuli ${ }^{60}$. A 3D cube is rendered for an observer viewing the screen (A) at right-angles, in the normal way, then (B-E) at progressively larger oblique angles: $10^{\circ}, 20^{\circ}, 35^{\circ}, 45^{\circ}$.

\section{REFERENCES}

$1 \quad$ Pinker, S. How the mind works. (The Penguin Press, 1997).

2 Schechner, Y. Y. \& Kiryati, N. Depth from defocus vs. stereo: How different really are they? Int J Comput Vision 39, 141-162, (2000).

3 Held, R. T., Cooper, E. A., O'Brien, J. F. \& Banks, M. S. Using Blur to Affect Perceived Distance and Size. Acm T Graphic 29, (2010).

Legge, G. E. Binocular contrast summation--I. Detection and discrimination. Vision Res 24, 373-383, (1984).

Changizi, M. A. \& Shimojo, S. "X-ray vision" and the evolution of forward-facing eyes. J Theor Biol 254, 756-767, (2008).

Read, J. C. A. Early computational processing in binocular vision and depth perception. Progress in biophysics and molecular biology 87, 77-108, (2005).

$7 \quad$ Hartley, R. \& Zisserman, A. Multiple view geometry in computer vision. (Cambridge University Press, 2000).

8 Cheong, L. F. \& Gao, Z. Quasi-Parallax for Nearly Parallel Frontal Eyes A Possible Role of Binocular Overlap During Rapid Locomotion. Int J Comput Vision 101, 45-63, (2013).

9 Read, J. C. A., Phillipson, G. P. \& Glennerster, A. Latitude and longitude vertical disparities. J Vis 9, 11 11-37, (2009).

10 Tweed, D., Haslwanter, T. \& Fetter, M. Optimizing gaze control in three dimensions. Science 281, 1363-1366, (1998).

11 Schreiber, K., Crawford, J. D., Fetter, M. \& Tweed, D. The motor side of depth vision. Nature 410, 819-822, (2001). 
Martin, G. \& Katzir, G. in 22nd International Ornithological Congress. (eds N.J. Adams \& R.H. Slotow) 2711-2728.

Rossel, S. Binocular stereopsis in an insect. Nature 302, 821-822, (1983).

Collett, T. Stereopsis in toads. Nature 267, 349-351, (1977).

van der Willigen, R. F. Owls see in stereo much like humans do. J Vision 11, 1-27, (2011).

Fox, R., Lehmkuhle, S. W. \& Bush, R. C. Stereopsis in the falcon. Science 197, 79-81, (1977).

Parker, A. J. Binocular depth perception and the cerebral cortex. Nature reviews. Neuroscience 8, 379-391, (2007).

Fielder, A. R. \& Moseley, M. J. Does stereopsis matter in humans? Eye 10 ( Pt 2), 233-238, (1996).

O'Connor, A. R., Birch, E. E., Anderson, S. \& Draper, H. The functional significance of stereopsis. Invest Ophthalmol Vis Sci 51, 2019-2023, (2010).

Melmoth, D. R., Finlay, A. L., Morgan, M. J. \& Grant, S. Grasping deficits and adaptations in adults with stereo vision losses. Invest Ophthalmol Vis Sci 50, 3711-3720, (2009).

Read, J. C. A., Begum, S. F., McDonald, A. \& Trowbridge, J. The binocular advantage in visuomotor tasks involving tools. $i-$ Perception 4, 101-110, (2013).

Ives, H. Airplane photography. (J. B. Lippincott Company 1920).

Julesz, B. Foundations of cyclopean perception. (University of Chicago Press, 1971).

Tyler, C. W. \& Clarke, M. B. in Stereoscopic Displays and Applications. (ed Proc. SPIE 1256).

D., P. J. in Visual Neurosci (eds J. D. Pettigrew, K. J. Sanderson, \& W. R. Lewick) Ch. 15, 208-222 (Cambridge University Press, 1986).

Collett, T. S. Vision: Simple stereopsis. Current Biology 6, 1392-1395, (1996).

van der Willigen, R. F., Frost, B. J. \& Wagner, H. Depth generalization from stereo to motion parallax in the owl. Journal of comparative physiology. A, Neuroethology, sensory, neural, and behavioral physiology 187, 997-1007, (2002).

van der Willigen, R. F., Harmening, W. M., Vossen, S. \& Wagner, H. Disparity sensitivity in man and owl: psychophysical evidence for equivalent perception of shape-from-stereo. J Vis 10, (2010).

Clarke, P. G., Donaldson, I. M. \& Whitteridge, D. Binocular visual mechanisms in cortical areas I and II of the sheep. The Journal of physiology 256, 509-526, (1976).

Timney, B. \& Keil, K. Local and global stereopsis in the horse. Vision Res 39, 1861-1867, (1999).

Smith, G. E. The Evolution of Man. (Oxford University Press., 1924).

Helmholtz, H. v. Handbuch der Physiologischen Optik. (Leopold Voss, 1867).

Cooper, E. A., Burge, J. \& Banks, M. S. The vertical horopter is not adaptable, but it may be adaptive. J Vis 11, (2011).

Mitchell, T. R. T. The Role of Binocular Vision in Mammalian Locomotion, Duke University, (2009).

Srinivasan, M. V. \& Davey, M. Strategies for Active Camouflage of Motion. P Roy Soc B-Biol Sci 259, 19-25, (1995).

Endler, J. A., Endler, L. C. \& Doerr, N. R. Great bowerbirds create theaters with forced perspective when seen by their audience. Current biology : CB 20, 1679-1684, (2010).

Wheatstone, C. On some remarkable, and hitherto unobserved, Phenomena of Binocular Vision. Philosophical Transactions of the Royal Society of London 128, 371 - 394, (1838).

Gombrich, E. Art and Illusion: A study in the psychology of pictorial representation., (Phaidon, 1997).

Gombrich, E. The image and the eye: further studies in the psychology of pictorial representation. (1982).

Secundus, C. P. Natural History. trans. Dr Philemon Holland (1847).

da Vinci, L. Notebooks. trans. Jean Paul Richter (1888).

da Vinci, L. Treatise on Painting. trans. John Francis Rigaud (J. B. Nichols \& son, 1835).

Kubovy, M. The psychology of perspective and Renaissance art. (Cambridge University Press, 1988).

Claparède, E. Stéréoscopie monoculaire paradoxale. Annales d'Oculistique 132, 465-466, (1904).

Arago, F. Fixation des images qui se forment au foyer d'une chambre obscure. Comptes-rendus hebdomadaries des Séances de l'Académie des Sciences Paris 8, 4-8, (1839).

Gombrich, E. in The image and the eye: further studies in the psychology of pictorial representation 40-62 (Phaidon, 1982).

Howson, S. Douglas Trumbull: The Quest for Immersion. Metro 169, 112, (2011).

Plato. Sophist. trans. Benjamin Jowett.

Ruppel, W., Alff, Y. \& Göllner, h. Study on the Acceptance of Higher Frame Rate Stereoscopic 3D in Digital Cinema. Society of Motion Picture and Television Engineers, 1-12, (2013).

Shibata, T., Kim, J., Hoffman, D. M. \& Banks, M. S. Visual discomfort with stereo displays: Effects of viewing distance and direction of vergence-accommodation conflict. Proc Spie 7863, 78630P78631-78630P78639, (2011).

Kim, J., Kane, D. \& Banks, M. S. Visual Discomfort and the Temporal Properties of the Vergence-Accommodation Conflict. Proc Spie 8288, 828811, (2012).

Lambooij, M. \& IJsselsteijn, W. Visual Discomfort and Visual Fatigue of Stereoscopic Displays: A Review. J Imaging Sci Techn 53, 030201-030201-030214, (2009). 
Yang, S. N. \& Sheedy, J. E. Effects of Vergence and Accommodative Responses on Viewer's Comfort in Viewing 3D Stimuli. Proc Spie 7863, (2011). Vis 11, (2011). 1707). and performance 13, 323-334, (1987).

58 Vishwanath, D., Girshick, A. R. \& Banks, M. S. Why pictures look right when viewed from the wrong place. Nature neuroscience 8, 1401-1410, (2005).

59 Pizlo, Z., Sawada, T., Li, Y., Kropatsch, W. G. \& Steinman, R. M. New approach to the perception of 3D shape based on veridicality, complexity, symmetry and volume. Vision Res 50, 1-11, (2010).

60 Hands, P., Smulders, T. V. \& Read, J. C. A. Stereoscopic 3D content appears relatively veridical when viewed from an oblique angle. J Vision in press, (2015).

61 Banks, M. S., Held, R. T. \& Girshick, A. R. Perception of 3-D Layout in Stereo Displays. Information display 25, 12-16, (2009).

62 Bereby-Meyer, Y., Leiser, D. \& Meyer, J. Perception of artificial stereoscopic stimuli from an incorrect viewing point. Perception \& psychophysics 61, 1555-1563, (1999).

63 Friedman, D. S. et al. Prevalence of amblyopia and strabismus in white and African American children aged 6 through 71 months the Baltimore Pediatric Eye Disease Study. Ophthalmology 116, 2128-2134 e2121-2122, (2009). Clifford, C. W. Binocular rivalry. Current biology : CB 19, R1022-1023, (2009).

65 Elliott, S. \& Shafiq, A. Interventions for infantile esotropia. The Cochrane database of systematic reviews 7, CD004917, (2013).

66 Stewart, C. E. et al. The effect of amblyopia treatment on stereoacuity. Journal of AAPOS : the official publication of the American Association for Pediatric Ophthalmology and Strabismus / American Association for Pediatric Ophthalmology and Strabismus 17, 166-173, (2013).

67 Knox, P. J., Simmers, A. J., Gray, L. S. \& Cleary, M. An exploratory study: prolonged periods of binocular stimulation can provide an effective treatment for childhood amblyopia. Invest Ophthalmol Vis Sci 53, 817-824, (2012).

68 Birch, E. E., Stager, D. R., Sr., Berry, P. \& Leffler, J. Stereopsis and long-term stability of alignment in esotropia. Journal of AAPOS : the official publication of the American Association for Pediatric Ophthalmology and Strabismus / American Association for Pediatric Ophthalmology and Strabismus 8, 146-150, (2004).

69 Birch, E. E. \& Stager, D. R., Sr. Long-term motor and sensory outcomes after early surgery for infantile esotropia. Journal of AAPOS : the official publication of the American Association for Pediatric Ophthalmology and Strabismus / American Association for Pediatric Ophthalmology and Strabismus 10, 409-413, (2006).

70 Drover, J. R., Stager, D. R., Sr., Morale, S. E., Leffler, J. N. \& Birch, E. E. Improvement in motor development following surgery for infantile esotropia. Journal of AAPOS : the official publication of the American Association for Pediatric Ophthalmology and Strabismus / American Association for Pediatric Ophthalmology and Strabismus 12, 136-140, (2008). Birch, E. E., Fawcett, S. \& Stager, D. R. Why does early surgical alignment improve stereoacuity outcomes in infantile esotropia? Journal of AAPOS : the official publication of the American Association for Pediatric Ophthalmology and Strabismus / American Association for Pediatric Ophthalmology and Strabismus 4, 10-14, (2000). Committee on, P., Ambulatory Medicine, S. o. O. A. A. o. C. O., American Association for Pediatric, O., Strabismus \& American Academy of, O. Eye examination in infants, children, and young adults by pediatricians. Pediatrics 111, 902-907, (2003).

73 Birch, E. E. \& Wang, J. Stereoacuity outcomes after treatment of infantile and accommodative esotropia. Optometry and vision science : official publication of the American Academy of Optometry 86, 647-652, (2009).

74 Adams, W. E., Leske, D. A., Hatt, S. R. \& Holmes, J. M. Defining real change in measures of stereoacuity. Ophthalmology 116, 281-285, (2009).

75 Alcala-Quintana, R. \& Garcia-Perez, M. A. A comparison of fixed-step-size and Bayesian staircases for sensory threshold estimation. Spatial vision 20, 197-218, (2007).

76 King-Smith, P. E., Grigsby, S. S., Vingrys, A. J., Benes, S. C. \& Supowit, A. Efficient and unbiased modifications of the QUEST threshold method: theory, simulations, experimental evaluation and practical implementation. Vision Res 34, 885-912, (1994). 